\title{
Fertilidade após a inseminação artificial intra- cervical ou laparoscópica intra-uterina de ovelhas utilizando diluidores à base de água de coco
}

\author{
Vanessa Porto MACHADO ${ }^{1}$ \\ José Ferreira NUNES ${ }^{1}$ \\ Airton Alencar de ARAÚJO ${ }^{1}$ \\ Dimas René Perez \\ FERNANDÉZ ${ }^{2}$ \\ Marina Albuquerque \\ CORDEIRO $^{1}$ \\ Cláudio Henrique Nogueira \\ de MEDEIROS ${ }^{3}$ \\ André Luíz Nogueira de \\ MEDEIROS $^{3}$ \\ Alexandre Weick Uchôa \\ MONTEIRO $^{3}$

\section{Correspondência para:} \\ VANESSA PORTOMACHADO \\ Laboratório de Tecnologia do Sêmen \\ Caprino e Ovino \\ Programa de Pós-graduação em Ciências \\ Veterinárias \\ Faculdade de Veterinária \\ Universidade Estadual do Ceará (UECE) \\ Av. Paranjana, 1700 - Campus do Itaperi \\ 60740 - 000 - Fortaleza-CE \\ v_porto@yahoo.com.br
}

Recebido para publicação: 13/04/2004 Aprovado para publicação: 12/12/2006

\author{
1 - Laboratório de Tecnologia do Sêmen Caprino e Ovino do Programa de \\ Pós-graduação em Ciências Veterinárias da Faculdade de Veterinária da \\ Universidade Estadual do Ceará, Fortaleza - CE \\ 2 - Genética e Produção de Caprinos e Ovinos, Quixadá - CE. \\ 3 - Associação de Agronegócios de Caprinos e Ovinos - ANOVIC, Maceió - AL
}

\section{Resumo}

O objetivo deste estudo foi avaliar a taxa de prenhez em ovelhas utilizando diluidores à base de água de coco (in natura: ACN ou em pó: ACP-102 ${ }^{\circledR}$ ) após inseminação artificial intra-cervical (Experimento 1) ou laparoscópica intra-uterina (Experimento 2). Experimento 1. Sêmen de 4 carneiros Santa Inês foi coletado, diluído a $37^{\circ} \mathrm{C}$ (T1: ACN; T2: ACP- $102^{\circledR}$ ) e resfriado á $4^{\circ} \mathrm{C} .56$ ovelhas Santa Inês foram inseminadas cervicalmente após a sincronização, 31 utilizando o diluidor ACN e 25 o diluidor ACP-102 ${ }^{\circledR}$. Experimento 2. Sêmen de 5 carneiros adultos (Santa Inês: $n=04$; Dorper $n=01$ ) foi coletado e processado de acordo com o descrito. 64 ovelhas SRD foram inseminadas intra-uterina por laparoscopia após a sincronização do estro, 37 utilizando o diluidor ACN e 27 o diluidor ACP-102 ${ }^{\circledR}$. O diagnóstico de gestação foi realizado por ultra-sonografia. A taxa de prenhez entre os tratamentos, dentro de cada método de inseminação, entre cada diluidor para ambos métodos de inseminação foi analisada pelo teste do Qui-quadrado $(\mathrm{p}<0,05)$. A taxa de prenhez após IA intra-cervical (ACN: 25,8\% vs. ACP-102 ${ }^{\circledR}: 48 \%$ ) e laparoscópica (ACN: $72,9 \%$ vs ACP-102 ${ }^{\circledR}: 70,3 \%$ ) não foi influenciada pelos diluidores. A taxa de prenhez foi influenciada pelo método de inseminação (intracervical: 35,7\% vs. laparoscopia: 71,8\%; $<<0,05)$. Os diluidores a base de água de coco podem ser utilizados nas inseminações artificiais cervical ou laparoscopica em ovelhas do Nordeste do Brasil em condições de campo.

\section{Introdução}

A criopreservação dos espermatozóides ovino teve um considerável progresso prático e econômico na aplicação da inseminação artificial (IA) em condições de campo ${ }^{1}$.

Muitos fatores podem reduzir a fertilidade no processo de IA: o escore corporal dos animais, a composição dos diluidores, o processo de resfriamento ${ }^{2}$, tempo da IA, a dose inseminante ${ }^{3}$ e o método de inseminação (intra-uterina, transcervical, vaginal) ${ }^{4}$.

$\mathrm{Na}$ inseminação cervical, um volume
Palavras-chave: Diluidor. Água de coco em pó. Inseminação intracervical. Inseminação laparoscopia intrauterina. Ovelhas. reduzido $(0,25 \mathrm{~mL})$ e uma concentração elevada de espermatozóides é necessária para a obtenção de fertilidade satisfatória. A laparoscopia requer um reduzido número de espermatozóides por dose, equipamentos caros e mão-de-obra especializada. A fertilidade é geralmente mais elevada quando este método é utilizado, pois o sêmen é depositado próximo do local de fecundação.

O diluidor deve ser capaz de proteger os espermatozóides durante o processo de criopreservação ${ }^{5}$. Os diluidores à base de leite desnatado para o sêmen resfriado $\mathrm{o}^{6,7}$ e o TRIS-ácido cítrico-glicose ${ }^{8}$ suplementado de 
gema de ovo para o sêmen congelado são amplamente utilizados com sucesso no processamento do sêmen ovino?.

O endosperma líquido do coco (água de coco) pode ser utilizada na criopreservação do sêmen de pequenos ruminantes ${ }^{10}$. O diluidor água de coco in natura (ACN) tem sido utilizado na criopreservação do sêmen de várias espécies, como: caprinos ${ }^{11,12}$, ovinos $^{13}$, bubalinos ${ }^{14}$, suínos ${ }^{15}$, caninos $^{16} \mathrm{e}$ humanos $^{10}$. O diluidor água de coco em pó (ACP) obtido da água do coco in natura após um processo de desidratação está sendo desenvolvido. Este diluidor já foi utilizado no resfriamento do sêmen caprino $\left(4^{\circ} \mathrm{C}\right)$ com resultados satisfatórios de fertilidade ${ }^{17}$. A água de coco em pó (ACP) poderá ser utilizada em regiões nas quais não exista $\mathrm{O}$ fruto do coqueiro. Todavia, o diluidor ACP ainda não foi utilizado no resfriamento do sêmen ovino para a inseminação artificial cervical ou laparoscópica. Para tal fim, a ACP teve seu $\mathrm{pH}$ e osmolsriade ajustadas para a espécie ovina e recebeu a denominação de ACP- $102^{\circledR}$.

Os objetivos deste estudo foram comparar a taxa de prenhez em condições de campo, após inseminação artificial cervical ou laparoscópica utilizando o sêmen ovino diluído em soluções à base de água de coco $\left(\right.$ ACN ou ACP-102 ${ }^{\circledR}$ ) e resfriado à $4^{\circ} \mathrm{C}$ e avaliar a viabilidade e a praticidade da utilização do diluidor ACP-102 ${ }^{\circledR}$.

\section{Material e métodos}

\section{Experimento 1 :}

Inseminação cervical utilizando sêmen ovino diluído em soluções à base de água de $\mathrm{coco}$

O experimento 1 foi realizado no município de Major Isidoro, Alagoas, situado na região Nordeste do Brasil ( $9^{\circ} 32^{\prime}$ latitude sul/ $36^{\circ} 59^{\prime}$ longitude oeste) teve como objetivo avaliar a eficiência dos diluidores à base de água de coco (ACN e ACP-102 ${ }^{\circledR}$ ) sobre a fertilidade de ovelhas Santa Inês após a IA cervical.

Os animais foram criados em sistema semi-intensivo. Os carneiros se alimentavam de pastagem nativa durante o dia e á noite, cada animal recebia $1 \mathrm{~kg}$ de concentrado. Às ovelhas foi fornecido $0,3 \mathrm{~kg}$ de concentrado. Todos os animais tinham acesso ao sal mineral e a água ad libitum.

Os diluidores (ACN; ACP-102 ${ }^{\circledR}$ ) foram preparados no dia da IA conforme descrito a seguir:

Diluidor ACN (T1): água de coco filtrada $(50 \mathrm{~mL})$; água destilada $(25 \mathrm{~mL})$, solução de citrato de sódio à $5 \%(25 \mathrm{~mL})$ e $3 \%$ de gema de ovo (pH: 6,5 - 6,7; 300 mOsm).

Diluidor ACP-102 ${ }^{\circledR}$ (T2): ACP-102 $2^{\hat{a}}$ $(12 \mathrm{~g})$, água destilada $(50 \mathrm{~mL})$, solução de citrato de sódio à 2,5\% (50 mL), e 3\% de gema de ovo ( $\mathrm{pH}: 6,6 ; 302 \mathrm{mOsm})$. A água de coco em pó (ACP) foi obtida utilizandose o equipamento "Spray Drying" em condições de temperatura e pressão controladas.

O sêmen de carneiros Santa Inês $(\mathrm{n}=4)$ com idade média de 12 meses e escore corporal mínimo de 3 (0-5) foi coletado durante o período seco (Agosto de 2002) utilizando a vagina artificial. Referidos carneiros foram previamente adaptados à coleta de sêmen utilizando a vagina artificial e tiveram o sêmen avaliado quanto à motilidade massal (MM), percentual de espermatozóides móveis (PEM), motilidade individual progressiva (vigor) e concentração espermática, estando todos os paraâmetros de acordo com o manual do Colégio Brasileiro de Reprodução.

Foram utilizados dois ejaculados de cada reprodutor com motilidade massal e motilidade individual progressiva $>4(0-5)$ e com um mínimo de $90 \%$ de espermatozóides móveis. O sêmen foi diluído à $37^{\circ} \mathrm{C}$ em ACN e ACP-102â, acrescentando em média $4,5 \mathrm{~mL}$ de diluidor para cada 1,0 mL de sêmen (diluição prática de 1:4,5). Em seguida, o sêmen foi resfriado a $4^{\circ} \mathrm{C}$ e armazenado a esta temperatura até o momento das inseminações, por um período máximo de seis horas.

Foram utilizadas ovelhas Santa Inês 
adultas $(\mathrm{n}=56)$ com idades variando de 1 à 4 anos e escore corporal mínimo de 3 (0-5). $\mathrm{O}$ estro foi sincronizado utilizando espojas intravaginais impregnadas com $50 \mathrm{mg}$ de acetato de medroxiprogesterona - MAP (Anestron ${ }^{\hat{a}}$, Química Santa Maria, RJ - Brasil) , que permaneceram na porçao cranial da vagina durante 14 dias. No dia da remoção das esponjas, em cada ovelha foram administrados 200 UI de gonadotrofina coriônica equina - eCG (Novormon ${ }^{\hat{a}}$, Syntex S.A., Argentina) por via intra-muscular.

As ovelhas foram inseminadas entre 48-50 h após a remoção das esponjas, utilizando sêmen diluído em ACN ( $\mathrm{n}=31)$ ou ACP-102 ${ }^{\circledR}(\mathrm{n}=25)$, depositando-se 0,25 $\mathrm{mL}$ em cada ovelha por via cervical.

\section{Experimento 2:}

Inseminação laparoscópica utilizando sêmen ovino diluído em soluções à base de água de coco

O experimento 2 foi realizado no município de Quixeramobim, Ceará, situado na região Nordeste do Brasil ( $8^{\circ} 28^{\prime}$ latitude sul $/ 62^{\circ} 3^{\prime}$ longitude oeste) com o objetivo de avaliar a eficiência de diluidores à base de água de coco (ACN e ACP-102 ${ }^{\circledR}$ ) sobre a fertilidade de ovelhas sem raça definida (SRD) após IA laparoscópica.

Os carneiros foram mantidos em um sistema intensivo de criação, enquanto as ovelhas permaneceram em sistema extensivo. Cada carneiro recebeu 0,6 kg de concentrado/dia. Todos os animais tinham acesso ao sal mineral e a água ad libitum.

Os diluidores (ACN e ACP-102 ${ }^{\circledR}$ ) foram preparados no dia da inseminação como descrito no experimento1.

$O$ sêmen de 5 carneiros adultos (Santa Inês: $: \mathrm{n}=04$; Dorper: $\mathrm{n}=01$ ) com idades média de 3 anos e escore corporal mínimo de 3 (0-5) foi coletado durante o período seco (Outubro, 2002) utilizando vagina artificial. Referidos carneiros foram previamente adaptados à coleta de sêmen utilizando a vagina artificial e tiveram o sêmen avaliado quanto à motilidade massal (MM), percentual de espermatozóides móveis (PEM), motilidade individual progressiva (vigor) e concentração espermática, estando todos os paraâmetros de acordo com o manual do Colégio Brasileiro de Reprodução.

Dois ejaculados de cada reprodutor com motilidade massal e individual progressiva $>3(0-5)$, e com um mínimo de $80 \%$ de espermatozóides móveis foram coletados no dia das inseminações. O sêmen foi diluído a $37^{\circ} \mathrm{C}$ em ACN ou ACP-102 ${ }^{\circledR}$, adicionando $9 \mathrm{~mL}$ de diluidor para cada 1,0 $\mathrm{mL}$ de sêmen (diluição prática 1:9), em seguida resfriado a $4^{\circ} \mathrm{C}$ e mantido a essa temperatura até o momento das inseminações artificiais.

Foram utilizadas ovelhas SRD adultas $(\mathrm{n}=64)$ com idade variando de 1 a 4 anos e escore corporal mínimo de 3 (0-5). O estro foi sincronizado conforme descrito no experimento 1. No dia da remoção das esponjas, em cada ovelha foram administrados 300UI de gonadotrofina coriônica equina - eCG (Novormon ${ }^{\circledR}$, Syntex S.A., Argentina) por via intra-muscular.

As ovelhas foram inseminadas por laparoscopia, entre 50-55h após a remoção das esponjas utilizando o sêmen diluído em $\mathrm{ACN}(\mathrm{n}=37)$ ou ACP-102 ${ }^{\circledR}(\mathrm{n}=27)$, o qual foi envasado em palhetas de $0,5 \mathrm{~mL}$, sendo 0,25 $\mathrm{mL}$ depositado em cada corno uterino.

O diagnóstico de gestação foi realizado aos 40 e 50 dias após as inseminações laparoscópica e cervical, respectivamente por ultra-sonografia (Falco Vet 100, Pie Medical, Netherlands) munido de sonda linear transretal de $5 \mathrm{mHz}$.

\section{Análise Estatistíca}

A taxa de prenhez ( $n^{\circ}$ ovelhas prenhes/ $n^{\circ}$ ovelhas inseminadas $x 100$ ) entre tratamentos (ACN ou ACP-102 ${ }^{\circledR}$ ) após inseminação cervical ou laparoscópica e dentro de cada diluidor entre os métodos de inseminação foram analisados pelo teste do Qui-quadrado a 5\% de significância.

\section{Resultados}

Os resultados dos experimentos $1 \mathrm{e}$ 
2 estão apresentados na tabela 1 . Um total de 120 ovelhas foram inseminadas. 56 ovelhas Santa Inês foram inseminadas cervicalmente (Experimento 1), das quais 31 utilizaram o diluidor $\mathrm{ACN}$ e 25 o diluidor ACP-102, resultando em taxas de fertilidade de $25,8 \%$ e $48 \%$, respectivamente. 64 ovelhas SRD adultas foram inseminadas por laparoscopia (Experimento 2), das quais 37 utilizaram o diluidor ACN e 27 o diluidor ACP-102, resultando em taxas de fertilidade de $72,9 \%$ e $70,3 \%$, respectivamente. A fertilidade não foi influenciada pelo diluidor utilizado quando as ovelhas foram inseminadas por via cervical ou laparoscópica respectivamente $(p>0,05)$. Os melhores resultados de fertilidade total foram obtidos quandos as ovelhas foram inseminadas por laparoscopia $(\mathrm{p}<0,05)$. A taxa de prenhez foi influenciada pelo diluidor utilizado entre os métodos de inseminação quando o diluidor ACN foi utilizado $(p<0,05)$.

\section{Discussão}

Os resultados deste estudo demonstraram um claro avanço na utilização de diluidores à base de água de coco após inseminação cervical ou laparoscópica em condições de campo, utilizando sêmen ovino resfriado $\left(4^{\circ} \mathrm{C}\right)$. O diluidor ACP-102 $2^{\hat{a}}$ preservou melhor a viabilidade espermática do que o diluidor ACN, principalmente após a inseminação artificial cervical.

No experimento 1 , o diluidor não influenciou a fertilidade, embora os melhores resultados tenham sido obtidos quando o diluidor ACP-102 $2^{\text {a }}$ foi utilizado. Resultados similares foram obtidos por Salgueiro et al. ${ }^{17}$ estudando os dois diluidores à base de água de coco na preservação do sêmen caprino resfriado à $4^{\circ} \mathrm{C}$ para a inseminação artificial cervical.

A baixa fertilidade quando utilizado o diluidor $\mathrm{ACN}$, deve-se provavelmente à obtenção de cocos de idades diferentes da padronizada (aproximadamente seis meses) para a produção deste diluidor, o que provavelmente influenciou a composição final do presente no diluidor, reduzindo assim a sua eficiência. A água de coco obtida de um fruto aos 6 meses de idade é uma solução natural ácida e estéril que contêm sais $(\mathrm{K}, \mathrm{Cl}, \mathrm{Na}, \mathrm{P}, \mathrm{Mg}, \mathrm{S}, \mathrm{Fe}, \mathrm{Cu})$, aminoácidos (glutâmico, serina, alanina, prolina, aminobutírico e outros), açúcares (monossacarídeos: glicose e frutose; dissacarídeo: sacarose), vitaminas e gorduras neutras $^{5}$. Segundo Watson ${ }^{14}$, o processo de resfriamento antecipa as modificações da membrana plasmática do espermatozóide e as células espermáticas uma vez capacitadas têm um curto período de vida e, se não forem expostas aos oócitos logo, não serão capazes de realizar a fertilização.

Os diluidores utilizados na preservação do sêmen ovino, geralmente possuem $\mathrm{pH}$ adequado $(6,8-7,0)$ e pressão osmótica fisiológica (300 $\mathrm{mOsm})$, protegendo assim os espermatozóides dos danos causados pelo choque térmico ${ }^{18}$. A falha na padronização na idade do coco utilizado para a produção do diluidor ACN pode também ter reduzido a fertilidade devido as alterações do $\mathrm{pH}$ e/ou osmolaridade deste, o que pode ter causado danos na membrana espermática e perda de motilidade. Com a motilidade reduzida e a estrutura peculiar da cérvice da ovelha $^{18}$, tornou-se mais difícil as células espermáticas menos viáveis alcançarem o local de fertilização (tuba uterina).

A fertilidade total após a inseminação cervical foi de $35,71 \%$. Resultados semelhantes foram observados por Naqvi et al. ${ }^{19}$ utilizando o sêmen congeladodescongelado diluido em TES-TRIS em ovelhas da raça Malpura em estro natural. Melhores resultados foram obtidos por Paulenz et al. ${ }^{9}$ em ovelhas mestiças da Noruega inseminadas cervicalmente em estro natural. A variação dos resultados de fertilidade entre os diferentes estudos devese provavelmente à diferença entre os animais utilizados, manejo no qual os animais foram submetidos ou a tecnologia do sêmen empregada. A fertilidade em ovelhas inseminadas cervicalmente com sêmen fresco mostrou resultados que variam de 40 - 
Tabela 1 - Taxa de prenhez de ovelhas deslanadas inseminadas por via cervical e laparoscópica utilizando sêmen ovino diluído em soluções à base deágua de coco

\begin{tabular}{lccc}
\hline & \multicolumn{3}{c}{ Taxa de prenhez $(\%)$} \\
Diluidores / Métodos de IA & $\mathrm{ACN}$ & $\mathrm{ACP}-102^{\circledR}$ & Total \\
\cline { 2 - 4 } Cervical & $25,8 \% \%^{\mathrm{a}, \mathrm{A}}$ & $48 \%{ }^{\mathrm{aA}}$ & $35,71 \%^{\mathrm{A}}$ \\
& $(8 / 31)$ & $(12 / 25)$ & $(20 / 56)$ \\
Laparoscópica & $72,9 \%{ }^{\mathrm{aB}}$ & $70,3 \%{ }^{\mathrm{aA}}$ & $71,8 \%{ }^{\mathrm{B}}$ \\
& $(27 / 37)$ & $(19 / 27)$ & $(46 / 64)$ \\
\hline
\end{tabular}

a,b: Diferentes letras subescritas indicam diferença significativa entre os diluidores, dentro de cada método de inseminação determinado pelo teste do Qui-quadrado $(p<0,05)$.

A,B: Diferentes letras subescritas indicam diferença significativa entre os métodos de inseminação, dentro de cada diluidor determinado pelo teste do Qui-quadrado $(\mathrm{p}<0,05)$.

ACN: água de coco in natura; ACP-102^: diluente para sêmen ovino à base de água de coco em pó.

Taxa de prenhez $=\left(n^{\circ}\right.$ ovelhas prenhes $/ n^{\circ}$ ovelhas inseminadas $\left.\times 100\right)$ a 40 e 50 dias após inseminação laparoscópica e cervical respectivamente.

$60 \%$ durante um curto perído de conservação (máximo 8 horas) (Eppleston e Maxwell ${ }^{5}$ ). A fertilidade após a IA cervical pode ser influenciada pelo local de deposição do sêmen no interior do trato genital feminino ${ }^{20}$, aumentando a taxa de fertilidade á medida que a profundidade da inseminação é aumentada ${ }^{18,20}$, podendo a fertilidade variar de $25,3 \%(0,5 \mathrm{~cm}$ de profundidade) para inseminação cervical rasa à $70,3 \%$ para a inseminação cervical profunda $(>4,0 \mathrm{~cm}$ de profundidade) (Salamon e Maxwell comunicações pessoais para Eppleston e Maxwell ${ }^{20}$ ).

No experimento 2, a fertilidade total após a inseminação laparoscópica em ovelhas sem raça definida foi de $71,8 \%$. O efeito do diluidor sobre a fertilidade não foi observado após a inseminação artificial laparoscópica, provavelmente devido ao fato de que as células espermáticas foram depositadas próximo ao local de ovulação o que aumenta a chance da fertilização ocorrer².

Semelhante fertilidade foi obtida entre os diluidores à base de água de coco utilizados neste experimento (ACN: 72,9\% versus ACP$102^{\circledR}: 70,3 \%$ ). A fertilidade observada neste experimento está de acordo com a observada por Eppleston e Maxwell ${ }^{20}$; Ghalsasi e Nimbkar ${ }^{21}$ após inseminação laparoscópica. Resultados semelhantes foram obtidos por Medeiros et al. ${ }^{9}$ utilizando o diluidor ACN nas mesmas condições de sincronização após a inseminação artificial laparoscópica em ovelhas sem raça definida em condições de campo. Câmara et al. ${ }^{22}$ obtiveram fertilidade de $41,38 \%$ utilizando o sêmen ovino diluído em TRIS-gema-glicerol e congelado na inseminação intra-uterina por via cervical. Logo, a fertilidade pode ser influenciada pelo diluidor $^{1}$, pelo processamento pelo qual o sêmen é submetido (resfriamento ou congelação), e pelo local de deposição do sêmen ${ }^{4}$.

A fertilidade total foi influenciada pelo método de inseminação utilizado, sendo os melhores resultados após a IA laparoscópica, resultados similares foram descritos por diferentes pesquisadores utilizando os diluidores : TRIS, Hepes, TRIS-prolina, TRISglicina - betaína, TRIS-glicina-prolina-betaína ${ }^{4}$; e leite desnatado + gema de ovo ${ }^{2}$ para sêmen congelado.

Em conclusão, os diluidores à base de água de coco podem ser utilizados em inseminações cervical e lapaproscópica. A laparoscopia foi o melhor método de inseminação, e o diluidor ACP-102 ${ }^{\circledR}$ parece ter preservado melhor a integridade das células espermáticas quando o método de inseminação cervical foi utilizado, entretanto outros estudos deverão ser realizados para certificar o seu mecanismo de proteção.

\section{Agradecimentos}

Os autores receberam apoio financeiro da Fundação Cearense de Apoio 


\begin{abstract}
ao Desenvolvimento Científico e ANOVIC, Maceió - AL e à Genética e Tecnológico - FUNCAP, Fortaleza-Ce. Os Produção de Caprinos e Ovinos Ltda, autores agradecem à Associação de Quixadá, Ceará pela colaboração na Agronegócio de Caprinos e Ovinos - condução deste experimento.
\end{abstract}

\title{
Fertility following intracervical or intrauterine laparoscopic insemination of ewes using extenders based on coconut water
}

\section{Abstract}

The objetive of this study was to evaluate pregnancy rates in ewes using extenders based on coconut water (in natura: $\mathrm{NCW}$ or in powder: PCW-102 ${ }^{\circledR}$ ) following intra - cervical (Trial 1) or intrauterine laparoscopic insemination (Trial 2). Trial 1. Semen from 4 Santa Inês rams were collected, diluted at $32^{\circ} \mathrm{C}\left(\mathrm{T} 1: \mathrm{NCW}\right.$; $\mathrm{T} 2$ : PCW-102 $\left.{ }^{\circledR}\right)$ and cooled to $4^{\circ} \mathrm{C}$. 56 adult Santa Inês ewes were inseminated intracervically following syncronization, 31 using the extender NCW and 25 the extender PCW-102 ${ }^{\circledR}$. Trial 2. Semen from 5 rams (Santa Inês: $\mathrm{n}=04$; Dorper $\mathrm{n}=01$ ) were collected and processed as described. 64 adult crossbred multiparous ewes were inseminated intrauterine by laparoscopy following oestrus syncronization. 37 ewes using the extender NCW and 27 the extender PCW- $102^{\circledR}$. The pregnancy diagnosis was performed by ultrasonography. The fertility rates between treatments for each method of insemination and within each extender for both methods were analysed by Chi-square test $(\mathrm{p}<0,05)$. The pregnancy rates following intra-cervical (NCW: $25.8 \%$ vs $\mathrm{PCW}-102^{\circledR}: 48 \%$ ) and intrauterine (NCW: $72.9 \%$ vs $70.3 \%$ ) inseminations were not influenced by the extenders. The pregnancy rate was influenced by insemination method (intra-cervical: $35.7 \%$ vs laparoscopy: $71.8 \% ; \mathrm{p}<0,05)$. The extenders based coconut water as basis can be used following cervical or intra-uterine laparoscopic inseminations in ewes from Northeast of Brazil under field conditions.

\section{Referências}

1 ALESSANDRO, A. G. D. et al. Post-thaw survival of ram spermatozoa and fertility after insemination as affected by prefreezing sperm concentration and extender composition. Theriogenology, v. 55, p. 11591170, 2001.

2 BYNE, G. P. et al. Effect of freezing rate of ram spermatozoa on subsequent fertility in vivo and in vitro. Animal Reproduction Science, v. 62, p. 265-275, 2000.

3 FERNANDÉZ-ABELHA, D.; PREVE, M. O.; VILLEGAS, $\mathrm{N}$. Insemination time and dilution rate of cooled and chilled ram semen affects fertility. Theriogenology, v. 60, p. 21-26, 2003.

4 SANCHÉZ - PARTIDA, L. G. et al. Fertility and its relationship to motility characteristics of spermatozoa in ewes after cervical, transcervcal, and intrauterine with frozen-thawed ram semen. Journal of Andrology, v. 2, p. 280-283, 1999.
Key-words:

Extender.

Powder coconut water. Intra-cervical insemination. Intrauterine laparoscopic insemination. Ewes.
5 NUNES, J. F.; CIRÍACO, A. L. T.; SUASSUNA, U. Produção e reprodução de caprinos e ovinos. Fortaleza: Editora gráfica, 1997. cap. 2, 199 p.

6 COLAS, G.; COUROT, M. Storage of ram semen. In: INTERNATIONAL CONGRESS WESTERN AUSTRALIA, 1976, Proceedings..., [S. I.: s. n.], p. 455465.

7 MARTIN, I. C. A. Diluents for the preservation of ram spermatozoa. I Diluents used at $7 \mathrm{oC}$ and $5 \mathrm{oC}$, contaning casein. Journal Biology Science, v. 19, p. 645 - 653, 1966.

8 SALAMON, S.; VISSER, D. Effect of composition of tris-based diluent and of thawing solution on survival of ram spermatozoa frozen by pellet method. Journal Biological Science, v. 25, p. 605-618, 1972.

9 PAULENZ, $\mathrm{H}$. et al. Effect of different extenders and storage temperatures on sperm viability of liquid ram semen, Theriogenology, v. 57, p. 823-836, 2002.

10 NUNES, J. F. Utilization of coconut water as extender 
of domestic animal and human semen. Revista Brasileira de Reprodução Animal, v. 22, p. 109-112, 1998.

11 NUNES, J. F. Coconut water as diluent for goat semen. In: INTERNATIONAL CONFERENCE IN GOATS, 1987 Brasília-DF, Proceedings... [S. I.: s. n.], 1987.

12 NUNES, J. F. Coconut water as extender for goat semen. Revista Científica, FCV - Luz, v. 3, p. 269-272, 1993.

13 MEDEIROS, A. L. N. et al. Insemination laparoscopic to field in mestizo sheep in the central interior of Ceará - preliminary data. Revista Brasileira de Reprodução Animal, v. 5, p. 84-86, 2002.

14 WATSON, P. F. Recent development and concept in the cryopreservation of spermatozoa and the assessment of their post-thawing function. Reproduction of Fertility and Development, v. 7, p. 871-891, 1995.

15 TONIOLLI, R.; MESQUITA, D. S. M. Fertility of sows inseminated with diluted semen in coconut water or B.T.S. extender. Revista Brasileira de Reprodução Animal, v. 14, p. 249-254, 1990.

16 UCHÔA, D. C.; CARDOSO, R. C. S.; SILVA, L. D. $M$. Artificial insemination with fresh semen in female Boxers using different extenders. Revista Brasileira de Reprodução Animal, v. 5, p. 150-152, 2002.

17 SALGUEIRO, C. C. et al. Utilization of extenders based on coconut water in natura and powder on the does artificial insemination at fixed time. Revista Brasileira de Reprodução Animal, v. 5, p. 96-98, 2002.

18 SALAMON, S.; MAXWELL, W. M. C. Storage of ram semen. Animal Reproduction Science, v. 62, p. 77- 111, 2000.

19 NAQVI, S. M. K. et al. Cervical penetration and trnscervical Al of tropical sheep (Malpura) at natural oestrus using frozen-thawed semen. Small Ruminant Research, v. 97, p. 329-333, 1998.

20 EPPLESTON, J.; MAXWELL, W. M. C. Recent attempts to improve the fertility of frozen ram semen inseminated into the cervix. Wool Technology of Sheep Breed., v. 3, p. 291- 302, 1993.

21 GHALSASI, P. M.; NIMBKAR, C. Evaluation of laparoscopic intrauterine insemination in ewes. Small Ruminant Research, v. 23, p. 69-73, 1996.

22 CÂMARA, D. R. et al. Transcervical artificial insemination with frozen-thawed semen in crossbred mairy sheeps. Revista Brasileira de Reprodução Animal, v. 5, p. 75-77, 2002.

23 VALLE, W. G. News on reproduction biotechnology in males. IN: WORD BUFFALO CONGRESS, 5., 1997, Caseria, Italy. Proceedings... [S. I.: s. n.], 1997. v. 1, p. 103-123. 